\title{
Reason for Treatment
}

National Cancer Institute

\section{Source}

National Cancer Institute. Reason for Treatment. NCI Thesaurus. Code C102702.

The reason the treatment was administered. 